\title{
Regularized Deblurring using Directional Prior with Sparse Representation
}

\author{
Subhajit Dhar, Vivek Maik, Mayank Srivastava
}

\begin{abstract}
Blind deconvolution defined as simultaneous estimation and removal of blur is an ill-posed problem that can be solved with well-posed priors. In this paper we focus on directional edge prior based on orientation of gradients. Then the deconvolution problem is modeled as L2-regularized optimization problem which seeks a solution through constraint optimization. The constrained optimization problem is done in frequency domain with an Augmented Lagrangian Method (ALM). The proposed algorithm is tested on various synthetic as well as real data taken from various sources and the performance comparison is carried out with other state of the art existing methods.
\end{abstract}

Keywords: Deblurring, Restoration, Sparse .prior, gradient angle prior.

\section{INTRODUCTION}

Recent increases in screen resolution of cameras, camcorders, and television has increased the need for image pre-processing in the form of high dynamic Range (HDR), color enhancement imaging (CEI), contrast enhancement and blur restoration. Out of these blur restoration offers the most difficult challenge as the solution finding is not direct and comes as in the form of an ill-posed problem. The ill-posed problem can be represented as

$$
y=H * x+n
$$

Here $y$ is the blurred imaging, $H$ is the blur kernel or point spread function (PSF) matrix and $n$ is the additive noise. Typically, in any image restoration given ' $y$ ' we have to find $H, x$ and $n$ which represent the unknown and ' $y$ ' represent the single known variable in the equation, so for finding 3 unknown variables a well-posed problem requires minimum use of atleast 3 equations. But here we have just single equation given in (1) which makes it an ill-posed problem. The ill-posed problem like the one above requires the use of mathematical methods known as optimization methods. In this paper we model the above given ill-posed problem as one such optimization method and solve it as

$$
\underset{x}{\operatorname{argmin}}\|y-H x\|^{2}+\lambda\|C x\|^{2}
$$

The above equation known as $L_{2}$-regularized minimization approach where the minimization happens for variable $x$. Through this minimization process we try to separate the variables $\mathrm{H}$ and $\mathrm{x}$ which otherwise appear as the convolution product $(*)$ in equation (1). The above equation when minimized for $x$ we will get $H$ which when applied to $y$ we will get imaging inverse solution.

Revised Manuscript Received on December 01, 2019

Subhajit Dhar, Department of Electronics and Communication Engineering, S.R.M. Institute of Science and Technology, Chennai, India.

Mayank Srivastava, Department of Electronics and Communication Engineering, S.R.M. Institute of Science and Technology,Chennai, India.

Vivek Maik, Department of Electronics and Communication Engineering, S.R.M. Institute of Science and Technology, Chennai, India.
The parameter $\lambda$ and $C$ represent the regularization parameter and high pass filter convergence of the algorithm.

There are various ways to enhance the abovementioned conventional approach. In this paper we propose the use of directional gradient orientation where the minimization process is controlled by weights decided according to the magnitude and direction of the gradient. This will give variation restoration which preserves edges better at different region decided by the gradient information at that particular location. The major contribution of the proposed deblurring algorithm can be listed as (i) Use of directional prior as constraint in the regularized optimization (ii) Use of alternating minimization to solve for $H$ and $x$, (iii) Use of penalty function as Bregman variables and Augmented Lagrangian for faster and better convergence. The rest of the paper is organized as follows : Section II explains the proposed algorithm, Section III explains noise in deblurring, Section IV explains Figures and Values, Section V explains the experimental results and Section VI concludes the paper.

\section{PROPOSED METHODOLOGY}

The image deconvolution / deblurring problem as optimization model described above is usually operated in the frequency domain as in frequency domain the convolution operation $(*)$ become multiplication in frequency and eases up on the computation process. The image deblurring also known as the deconvolution is the reverse of convolution where we separate the convolved variables $\mathrm{H}$ and $\mathrm{x}$ thereby separating the blur from the original sharp image. The end product of typical deblurring has estimated input sharp image and blur kernel as shown in (3). The deblurring does its best to remove the mathematical operators connecting $H, x$ and $n$ and give them as separate variables.

$$
y=H * x+n=H, x, n
$$

where $H$ denotes an unknown blur or kernel or point spread function which usually smaller in size $(3 \times 3$ to $13 \times 13)$ in our approach and $n$ is the additive noise in the observation.

Operator * stands for linear time domain convolution operator which includes shifting, multiplying and addition of $H$ and $x$. If we approximate $H$ to some value, we can deconvolve the image using

$$
x=H^{-1} y
$$

Where $x, H, y$ denotes frequency domain operators. 


\section{Regularized Deblurring using Directional Prior with Sparse Representation}
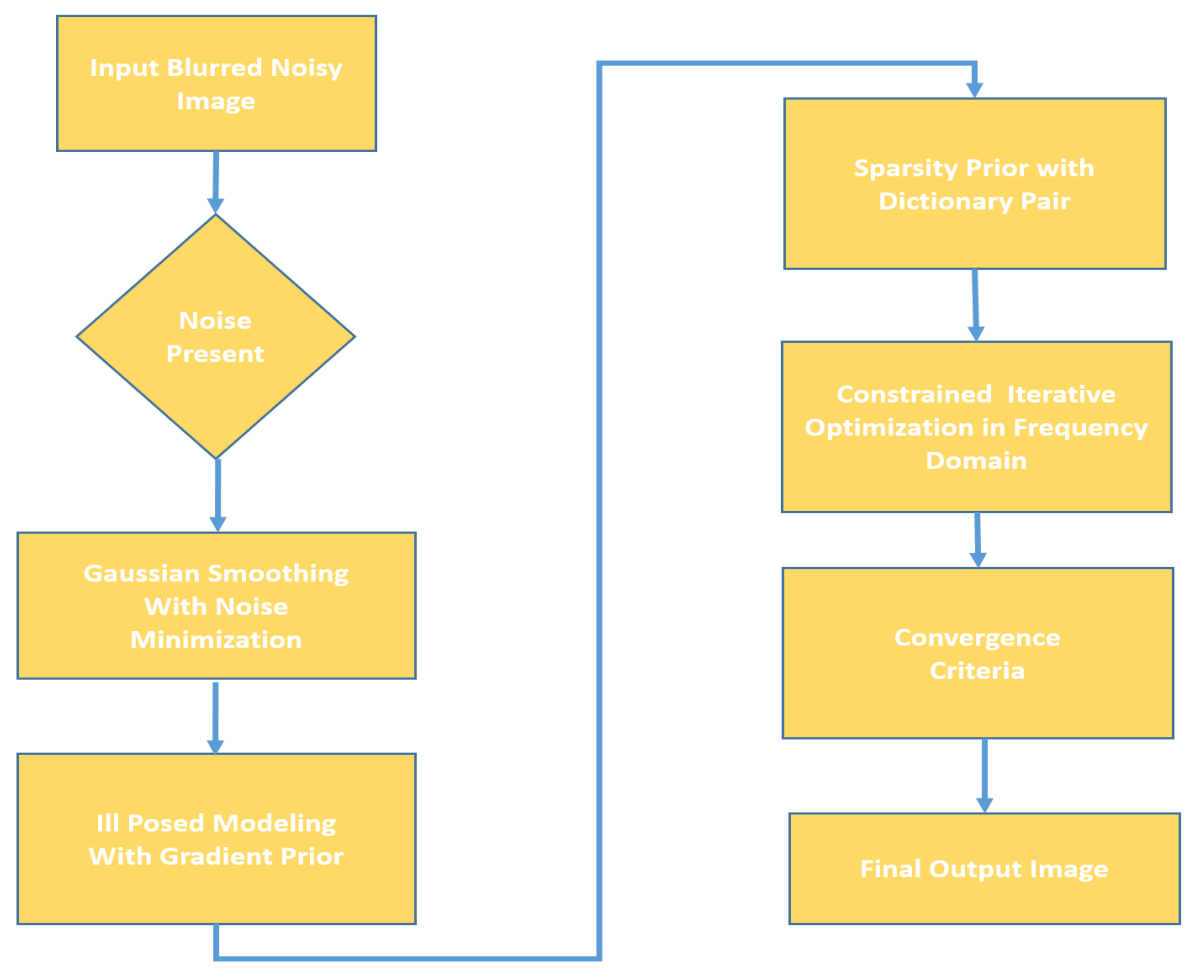

Fig.1. Block Diagram of the Proposed Deblurring Algorithm

The above solution is also not fixed because the inverse of a matrix doesn't exist always and step the results of deconvolution would be better if $H$ can be approximated in multiple steps. There we propose a multi-step deconvolution in multiple steps using directional gradient prior mathematically. The directional gradient is seamlessly integrated in to the least square optimization algorithm given in (2) and is solved iteratively with the directional gradients also contributing at each iteration. Using that we can approximate the solution as

$$
\hat{x}=\|y-H x\|^{2}+\lambda\|\nabla x y\|^{2}
$$

where $\lambda$ is the regularization term that controls to certain extent the degree of blurring thereby preserving the edges and smooth region. In this paper we boost the regularization performance with directional gradient as given in (5). Typical approaches used regularization parameters on a high frequency contents of the image but replacing it with gradient has given us better performance.

The inverse problem can be solved better by adopting direction prior regularization and multi-step $H$ estimation.

$$
\min \left\|y_{k}-H_{k} x\right\|_{2}^{2}
$$

Once the regularization term is modified with the gradient priors our next major contribution was to split the minimization step in (6) in to two step minimization problem. Using this two-step minimization, we keep two sets of control parameter that will be used consecutively but the first set of parameter controls the $\mathrm{x}$ variable and the second set of parameters controls the $\mathrm{H}$ parameter. The approach we call it as two step minimization problem. The above minimization term represents multistep optimization problem. For multistep optimization we have to do it with alternating minimization for $H$ and $x$. Since $H$ and $x$ are unknown for each step the value of $H$ and $x$ are modified alternatively along with other parameters.

$$
\begin{gathered}
x-\text { step } \min _{x}\{F\{x\}, V\{H\}\}+Q(x) \\
H-\text { step } \min _{H}\{F\{x\}, V\{H\}\}+R(x)
\end{gathered}
$$

Here $Q(x)$ and $R(h)$ represent the Bregman Variables. The global minimum of the above problem can be solved efficiently by also incorporating the Lagrangian penalty function as Augmented Lagrangian Variable given by

$$
\|y-H x\|_{2}^{2}+\sum_{n=1}^{M} \lambda_{n}\left\|\nabla_{\theta_{n}}-p_{n}\right\|^{2}
$$

where the summation term represent the number of gradient angles used. The proposed approach made use of 5 angles $[0,45,90,135,180]$ in the given order and $p_{n}$ represent the respective Augmented Lagrangian penalty terms. Through experimental trials it was observed that keeping separate value for the regularization with each angle gave better Signal to Noise Ratio (SNR) improvements that keeping an single regularization term for all the gradient angles. The values of the regularization was chosen in such a way that the gradients in horizontal $\left(0^{0}\right)$, vertical $\left(90^{\circ}\right)$ and diagonal $\left(45^{\circ}\right)$ were given more weightage as these edge details are more prominent in most of the cases. The other 2 angles were given weightage towards the latter part of the iterative minimization. 
The regularization part in (9) can be worked better by introducing sparse prior along with gradient prior. The sparsity prior in $\mathrm{L}_{2}$ norm will make sure the deblurred image don't have any speckle noise which is usually very prominent with more number of iterations or excessive optimization. Since the convergence on the iterative optimization for $\mathrm{H}$ and $\mathrm{x}$ is not always guaranteed and more than often the algorithm stops after a predefined number of iterations. When this happens at times the deblurring will lead to speckle noise which can be overcome by introducing sparsity prior to the above deblurring with gradient prior.

$$
D_{\alpha}\left[\|y-H x\|_{2}^{2}+\sum_{n=1}^{M} \lambda_{n}\left\|\nabla_{\theta_{n}}-p_{n}\right\|^{2}\right]
$$

where $D_{\alpha}$ represent the dictionary sparsity prior which will in introduced to $H$ and $x$ during optimization. This dictionary sparsity prior acts more like a noise filter by getting rid of high frequency spots / speckles that get introduced during the minimization process

\section{NOISE IN DEBLURRING}

In the above section we proposed a sparsity prior which helps in getting rid of high frequency speckles which get introduced during deblurring. But from (1) we have additive noise $n$ which also could affect the noise in deblurring. The algorithm classifies the image as either noisy or noiseless and the algorithm will perform the optimization in two steps as shown below. In the presence of noise, we assume a given distribution for the noise formation.

$$
y=H^{*} x+n
$$

In the presence of noise $n$, the above algorithm works the same except we need to do pre-processing to take care of the noise for $\mathrm{H}$ and $\mathrm{x}$ of size $\mathrm{MXN}$. In this paper we use a simple noise reduction method which use Gaussian smoothing to suppress the noise and then later this Gaussian smoothing is removed in the iterative deblurring stages. Then the deblurring model is given by

$$
\underset{x}{\arg \min }\left(\|y-H * x\| * G_{k}-\|y-H * x\|\right)
$$

In the above equation $G_{k}$ represents the Gaussian smoothing kernel and $\|y-H * x\|$ represents the noise $\mathrm{n}$. The minimization of 15 will take care of any small noise present in the input image initially prior to the deblurring. As the focus of this paper is deblurring we have not explored this area in detail.

\section{FIGURES \& VALUES}

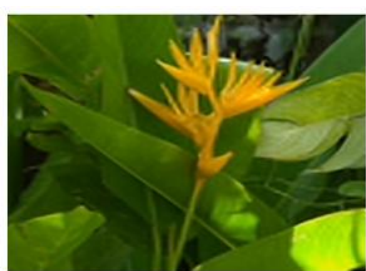

(a)

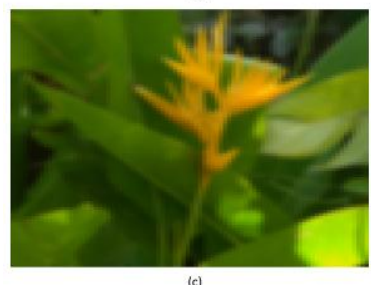

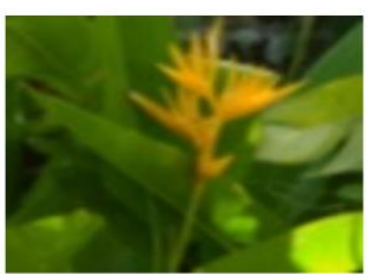

(b)

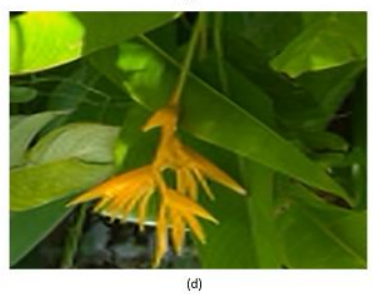

Fig. 2 : The experimental results of the proposed algorithm compared with various other methods [3], the PSNR values for images (From top: method1 PSNR=27.45dB, method2 PSNR= 25.32dB, method 3 PSNR=26.56dB, reconstructed image using proposed method PSNR 30.12dB)
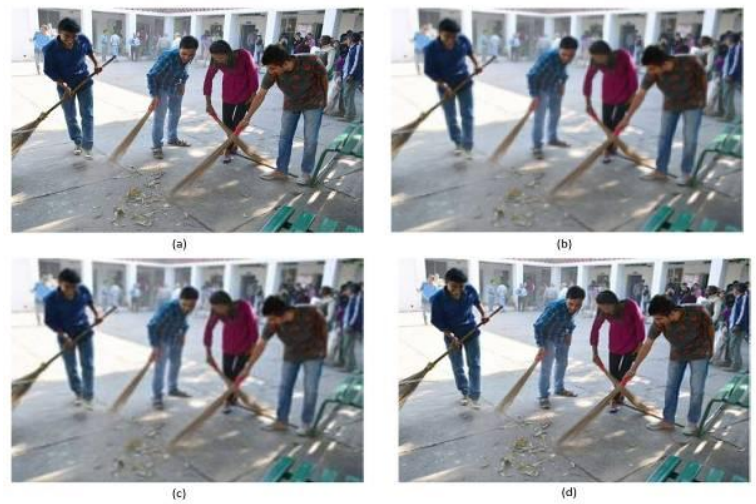

Fig. 3 : Real data set with experimented results. (a) captured image, (b) blurred image, (c) noisy image, (d) proposed algorithm result.

\section{EXPERIMENT RESULTS - TABLES}

Although image contents vary from image to image in order to demonstrate convergence properties of the proposed algorithm, the PSF estimates with respect to the Peak Signal to Noise Ratio (PNSR) and Structural Similarity Index (SSIM) of images. The blurred image compares the quality and standard values to systematic results. The PSF results compared with the estimated results for better deblurred images. We experimented two sets of data sets. The first data set were blurred using Uniform simulated blur and the second data set were blurred using Gaussian blur. For both blur noise variance of $\sqrt{2}$ was used. Figs 2 and Fig 3 show the image results of the proposed algorithm on captured image. Table I to IV show the PSNR and SSIM comparison result of the proposed method with standard state of the art methods such as FISTA, L0-SPAR, IDD-B3D, ASDS-REG, NCSR for various images available as open source dataset. The entire coding simulation was done in MATLAB platform.

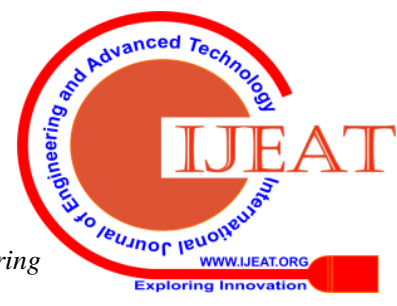


Regularized Deblurring using Directional Prior with Sparse Representation

TABLE I : PSNR COMPARISON BETWEEN VARIOUS ALGORITHMS: FISTA [12], L0-SPAR [ 10], IDD-BMD [09], ASDS [ 11] WITH PROPOSED METHOD FOR UNIFORM BLUR OF 9X9 SIZE WITH NOISE VARIANCE OF $\sqrt{2}$ FOR ONE SET OF IMAGES

\begin{tabular}{|c|c|c|c|c|c|c|c|c|c|c|}
\hline & Butterfly & Boats & Cameraman & House & Parrot & Lena & Barbara & Starfish & Peppers & Leaves \\
\hline FISTA & 28.37 & 29.04 & 26.82 & 31.99 & 29.11 & 28.33 & 25.75 & 27.75 & 28.43 & 26.49 \\
\hline LO-SPAR & 27.1 & 29.86 & 26.97 & 32.98 & 29.34 & 28.72 & 26.42 & 28.11 & 28.66 & 26.3 \\
\hline IDD-BM3D & 29.21 & 31.2 & 28.56 & 34.44 & 31.06 & 29.7 & 27.98 & 29.48 & 29.62 & 29.38 \\
\hline ASDS-Reg & 28.7 & 30.8 & 28.08 & 34.03 & 31.22 & 29.92 & 27.86 & 29.72 & 29.48 & 28.59 \\
\hline Proposed & 29.1 & 30.8 & 28.56 & 35.1 & 31.8 & 30.1 & 28.1 & 30.2 & 29.78 & 29.2 \\
\hline
\end{tabular}

TABLE II : PSNR COMPARISON BETWEEN VARIOUS ALGORITHMS: FISTA [12], L0-SPAR [ 10], IDD-BMD [09], ASDS [ 11] WITH PROPOSED METHOD FOR UNIFORM BLUR OF 9X9 SIZE WITH NOISE VARIANCE OF $\sqrt{2}$ FOR ANOTHER SET OF IMAGES

\begin{tabular}{|c|c|c|c|c|c|c|c|c|c|c|}
\hline & Butterfly & Boats & Cameraman & House & Parrot & Lena & Barbara & Starfish & Peppers & Leaves \\
\hline FISTA & 30.36 & 29.36 & 26.81 & 31.5 & 31.23 & 29.47 & 25.03 & 29.65 & 29.42 & 29.36 \\
\hline LO-SPAR & 30.73 & 31.68 & 28.17 & 34.08 & 32.89 & 31.45 & 27.19 & 31.66 & 29.99 & 31.4 \\
\hline IDD-BM3D & 29.83 & 30.27 & 27.29 & 31.87 & 32.93 & 30.36 & 27.05 & 31.91 & 28.95 & 30.62 \\
\hline ASDS-Reg & 29.83 & 30.27 & 27.29 & 31.87 & 32.93 & 30.36 & 27.05 & 31.91 & 28.95 & 30.62 \\
\hline Proposed & 30.21 & 30.94 & 28.2 & 32.12 & 33.67 & 31.02 & 28.14 & 32.65 & 29.48 & 30.94 \\
\hline
\end{tabular}

TABLE III : SSIM COMPARISON BETWEEN VARIOUS ALGORITHMS: FISTA [12], L0-SPAR [ 10], IDD-BMD [09], ASDS [ 11] WITH PROPOSED METHOD FOR GAUSSIAN BLUR OF 9X9 SIZE WITH NOISE VARIANCE OF $\sqrt{2}$ FOR ONE SET OF IMAGES

\begin{tabular}{|c|c|c|c|c|c|c|c|c|c|c|c|}
\hline & Butterfly & Boats & Cameraman & House & Parrot & Lena & Barbara & Starfish & Peppers & Leaves \\
\hline FISTA & 0.9119 & 0.8858 & 0.8627 & 0.9017 & 0.9002 & 0.8798 & 0.8375 & 0.8775 & 0.8813 & 0.8958 \\
\hline LO-SPAR & 0.8879 & 0.9094 & 0.8689 & 0.9225 & 0.9262 & 0.9063 & 0.8691 & 0.8951 & 0.9066 & 0.8776 \\
\hline IDD-BM3D & 0.9287 & 0.9304 & 0.9007 & 0.9369 & 0.9364 & 0.9197 & 0.9014 & 0.9167 & 0.92 & 0.9295 \\
\hline ASDS-Reg & 0.9053 & 0.9236 & 0.895 & 0.9337 & 0.9306 & 0.9256 & 0.9088 & 0.9208 & 0.9203 & 0.9075 \\
\hline Proposed & 0.9145 & 0.9562 & 0.924 & 0.9564 & 0.9417 & 0.9356 & 0.921 & 0.9348 & 0.9423 & 0.9186 \\
\hline
\end{tabular}

TABLE IV : SSIM COMPARISON BETWEEN VARIOUS ALGORITHMS: FISTA [12], L0-SPAR [ 10], IDD-BMD [09], ASDS [ 11] WITH PROPOSED METHOD FOR GAUSSIAN BLUR OF 9X9 SIZE WITH NOISE VARIANCE OF $\sqrt{2}$ FOR ANOTHER SET OF IMAGES

\begin{tabular}{|c|c|c|c|c|c|c|c|c|c|c|}
\hline & Butterfly & Boats & Cameraman & House & Parrot & Lena & Barbara & Starfish & Peppers & Leaves \\
\hline FISTA & 0.9452 & 0.9024 & 0.8845 & 0.8968 & 0.929 & 0.9011 & 0.8415 & 0.9256 & 0.9057 & 0.9393 \\
\hline LO-SPAR & 0.9442 & 0.9426 & 0.9136 & 0.9359 & 0.9561 & 0.943 & 0.8986 & 0.9496 & 0.9373 & 0.9512 \\
\hline IDD-BM3D & 0.9126 & 0.9064 & 0.8637 & 0.8978 & 0.9576 & 0.9058 & 0.8881 & 0.9491 & 0.9039 & 0.9304 \\
\hline ASDS-Reg & 0.9381 & 0.9371 & 0.9078 & 0.9333 & 0.9587 & 0.9389 & 0.9088 & 0.9551 & 0.9331 & 0.9508 \\
\hline Proposed & 0.9415 & 0.9463 & 0.9154 & 0.9478 & 0.9652 & 0.9438 & 0.9184 & 0.962 & 0.9414 & 0.9586 \\
\hline
\end{tabular}

\section{CONCLUSION}

Through this work we have proposed an image deblurring algorithm with the following contribution:

Use of sparsity prior, use of gradient angle prior, Lagrangian penalty with Bregman variables for better convergence, effective treatment of noise in the given ill posed equation. This method gives stability of image restoration by directional priori information. The sparse representation based upon PSNR estimated values under the assumption of SSIM results. The performance of the algorithm was found to be same as or better than some of the standard established algorithms found in recent literature. One drawback that was found during the execution of this project is the parameter values tuning which could be really cumbersome with so many prior. Next stage we are working on an adaptive algorithm that would take care of the parameter values through computational approach rather than manual calculation and tuning. 


\section{REFERENCE}

[1] Filip Sroubek and PeymanMilanfar., "Robust Multichannel Blind Deconvolution via Fast Alternating Minimization”. IEEE tran. Image Processing, vol. 21, no. 4, April 2012.

[2] Zhang Hongyinget. al., "Variational Image Deblurring Using Modified Hopfield Neural Network". IEEE 2006.

[3] F. Guichard, L. Moisan, and J. M. Morel, "A Review of PDE Models in Image Processing and Image Analysis," Journal de Physique IV,France. Vol. 12, pp. 1-18, 2002.

[4] Jain Feng Caiet.al., "Blind motion deblurring from a single image using sparse approximation". IEEE paper, 2009.

[5] J. Cai, S. Osher, and Z. Shen. "Linearized bregman iterations for frame-based image deblurring". UCLA CAM Reports (08-52), 2008.

[6] N. Joshi, R. Szeliski, and D. Kriegman. PSF estimation usingsharp edge prediction. In CVPR, 2008

[7] Zhaosong Lu, Yong Zhang., "An augmented Lagrangian approach for sparseprincipal component analysis". Mathematical optimization society 2011.

[8] GuoW.Wei., "Generalized Perona-Malik Equation for Image Restoration". IEEE signal processing letters, vol.6, no.7,july 1999.

[9] Carlos Bazan and Peter Blomgen., “ Image Smoothing and Edge Detection by Nonlinear Diifusion and Bilateral Filter". Digital processing conference, 2009.

[10] SarmilaPadhy and Ratnakar Dash., "Improved Spatilly Adaptive Denoising Algorithm to Suppress Gaussion Noise in an Image". International Journal of Computer Applications, vol. 67, no. 17, April 2013.

[11] Neel Joshi, Richard Szeliski, and David J.Krieman., "PSF estimation using Sharp Edge Prediction", 2009.

[12] R. Fegus et al. Removing camera shake from a single photograph ACM Transactions on Graphics, 27(3):787-794, August 2006.

[13] Y. Tai, H. Du, M. S. Brown, and S. Lin. "Image/video deblurringusing a hybrid camera". In CVPR, 2008.

[14] A. Gupta, N. Joshi, C.L. Zitnick, M.F. Cohen. "Single Image Deblurring using Motion Desity functions. IN ECCV, pages 171-184, 2010.

[15] TomerPeleg and Michael Elad. "A Statistical Prediction Model based on Sparse Representation for Single Image Super-Restoration". IEEE trans. On image processing, vol. 23, no. 6, June 2014.

[16] T. Peleg, Y. C. Eldar, and M. Elad, "Exploiting statistical dependencies in sparse representations for signal recovery," IEEE Trans. Signal Process., vol. 60, no. 5, pp. 2286-2303, May 2012.

[17] J. Yang, J. Wright, T. Huang, and Y. Ma, "Image super-resolution as sparse representation of raw image patches," in Proc. IEEE Conf. Comput. Vis. Pattern Recognit., Jun. 2008, pp. 1-8.

[18] Naoto Katsumata, Yasuo Matsuyama." Similar -Image Retrival Systems Using ICA and PCA Bases”. IJC on neural Netwoks, Montreal, Canada, August 2005.

[19] Haichao Zhang, David Wipf and Yanning Zhang. "Multi Image Blind Deblurring Using a Coupled Adaptive Sparse Prior". IEEE on Computer Vision \& Pattern Recognition. 2013.

[20] D. P.Wipf, B. D. Rao, and S. S. Nagarajan. Latent variable Bayesian models for promoting sparsity. IEEE Trans. on Information Theory, 57(9):6236-6255, 2011.

[21] G. Giannakis and R. Heath, "Blind identification of multichannel FIR blurs and perfect image restoration," IEEE Trans. Image Process., vol. 9, no. 11, pp. 1877-1896, Nov. 2000.

[22] H.-T. Pai and A. Bovik, "On eigenstructure-based direct multichannel blind image restoration," IEEE Trans. Image Process., vol. 10, no. 10, pp. 1434-1446, Oct. 2001. .

[23] M. Haindl and S. Šmberová, "Model-based restoration of short-exposure solar images," in Frontiers in Artificial Intelligence and Applications L. Jain and R. Howlett, Eds. Amsterdam, The Netherlands: IOS Press, 2002, pp. 697-706.

[24] V. Katkovnik, D. Paliy, K. Egiazarian, and J. Astola, "Frequency do-main blind deconvolution in multiframe imaging using anisotropic spa-tially-adaptive denoising," in Proc. 14th EUSIPCO, Sep. 2006, pp. 1-5.

[25] G. Panci, P. Campisi, S. Colonnese, and G. Scarano, "Multichannel blind image deconvolution using the bussgang algorithm: Spatial and multiresolution approaches," IEEE Trans. Image Process., vol. 12, no.11, pp. 1324-1337, Nov. 2003.

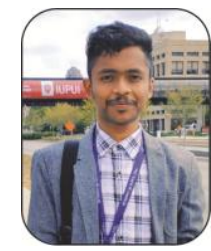

\section{AUTHORS PROFILE}

Subhajit Dhar is a student in the Department of Electronics And Communication Engineering at S.R.M. Institute of Science and Technology, Kattankulathur, Chennai. Associated with projects at IIT Madras and multiple ongoing projects through collaborations with Professors Internationally. A Scholarship holder from the same University. Several Training Experiences and internships nationally. Membership at IEI. Attended multiple conferences across India and Internationally at USA. Received Best Paper Award at the International Conference held at Indianapolis, Indiana, USA.

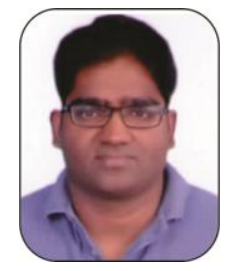

Dr. Vivek Maik is a Research Assistant Professor with the SRM Institute of Science and Technology, Chennai, India. He hold nine International patents and has more than 50 peer-reviewed publications during his academic career. His research interest includes signal, image and video processing with special focus on ill-posed, sparsity coding and optimization techniques. He is an active member of the Consumer Electronics Society and Indian Science Congress. He is also reviewer for several IEEE and Elsevier Journals \& Conferences.

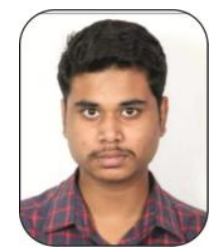

Mayank Srivastava studying Electronic and Communication Engineering from SRM institute of science and technology. I am currently working on various machine learning projects. I am always ready to learn new things. 